\title{
Perceptions of carotenoid and melanin colouration in faces among young Australian adults
}

\author{
Abstract \\ Objective: Human skin colour is influenced by three pigments: haemoglobin, carotenoids and melanin. Carotenoids are \\ abundant in fruits and vegetables, and when consumed accumulate in all layers of the skin, predominantly imparting \\ yellowness $\left(b^{*}\right)$. This study investigated the effect of the manipulation of carotenoid-based skin colour, relative to the \\ skin colour conferred by melanin on the perceptions of health amongst a group of Australian adults. \\ Method: Fifty-seven participants ( $\mathrm{n}=4$ male; mean age $27.9 \pm 7.5$ years) completed three computer-based experiments \\ on 50 trial faces. In the first two experiments, face image colour was manipulated along one or two independent single \\ carotenoid or melanin axes on each trial to "make the face appear as healthy as possible". In the third trial, face colour \\ was manipulated on both the carotenoid and melanin axes simultaneously. Results: For the single axis, participants \\ significantly increased melanin colouration and added carotenoid colouration to facial images that were initially low in \\ skin yellowness $\left(\mathrm{b}^{*}\right)$. When carotenoid and melanin axes were simultaneously manipulated, carotenoid colouration was \\ raised ( $\triangle \mathrm{E} 3.15$ ( $\mathrm{SE} \pm 0.19)$ and melanin colouration was lowered $\Delta \mathrm{E}-1.04(\mathrm{SE} \pm 0.1)$. Conclusions: Young Australian \\ adults perceive facial skin colouration, associated with both carotenoid intake from fruit and vegetables and melanin \\ due to sun exposure as conveying the appearance of health in young adults. However carotenoid colouration was more \\ important to health perception.
}

Key words: appearance, carotenoids, fruit and vegetables, healthy, melanin, perception

\section{Key points:}

1. The consumption of fruits and vegetables leads to the build-up of dietary carotenoids in human skin and along with melanin, contributes to skin colour yellowness.

2. Young Australian adults do not meet the Australian Dietary Guidelines for fruit and vegetables that recommend two serves of fruit and five serves of vegetables per day. They have one of the lowest intakes of fruits and vegetables compared to older age groups. 
3. Previous research suggests that humans have a preference for yellow pigmentation of skin. This study aimed to investigate the perceptions related to health with carotenoid based skin colour compared with melanin colouration in a group of young Australian adults.

\section{Introduction}

Human skin colour is determined by three major pigments. Haemoglobin contributes to red colouration, melanin which darkens and yellows the skin colour and carotenoids which contribute predominantly to skin yellowness (Alaluf, Heinrich, Stahl, Tronnier, \& Wiseman, 2002). Carotenoids are lipid soluble, red, orange and yellow pigments, abundant in many fruits and vegetables. Following fruit and vegetable consumption, these pigments accumulate in all layers of human skin (Alaluf et al., 2002; Mayne et al., 2010).

Fruit and vegetable consumption is related to the skin yellowness $\left(b^{*}\right)$ axis of the CIE $\mathrm{L}^{*} \mathrm{a}^{*} \mathrm{~b}^{*}$ colour space, where $\mathrm{L}^{*}$ represents skin lightness and positive values of $a^{*}$ and $b^{*}$ represents degrees of redness and yellowness respectively (Pezdirc et al., 2015; Stephen, Coetzee, \& Perrett, 2011). Self-reported increases in fruit and vegetable intake over a six -week period have shown to be associated with a measurable increase in skin yellowness ( $\left.b^{*}\right)$ and redness (a*) (Whitehead, Re, Xiao, Ozakinci, \& Perrett, 2012).

Skin colour due to carotenoid content has been shown to affect young adults' perceptions of an individual's overall appearance of health (Stephen et al., 2011; Stephen, Law Smith, Stirrat, \& Perrett, 2009; Whitehead, Ozakinci, \& Perrett, 2012). Recent studies using colour calibrated Caucasian facial images have shown that greater yellowness (b*) and lightness $\left(\mathrm{L}^{*}\right)$ is perceived by Scottish adults as conveying a more healthy (Stephen et al., 2011; Stephen et al., 2009; Whitehead, Ozakinci, et al., 2012) and attractive appearance (Lefevre, 2015). These results suggest that humans may express a preference for skin colour associated with higher intakes of fruit and vegetables (carotenoids), rather than skin colour associated with sun exposure (melanin) (Stephen et al., 2011; Stephen et al., 2009; Whitehead, Ozakinci, et al., 2012). Although this relationship has been investigated in other ethnicities including black South Africans and Asians (Stephen et al., 2011) it has not been investigated in an Australian population where sun exposure is common. 
In Australia, young adults perceive sun tanned skin to be healthy and attractive compared to untanned white skin (Hamilton et al., 2012). In addition, young Australian adults have one of the lowest intakes of fruit and vegetables compared to other adult life stages with only $40.6 \%$ those aged $19-30$ years meeting the guidelines of two servings of fruit and 2.9\% meeting five servings of vegetables per day (Australian Bureau of Statistics, 2014).

Therefore, the aim of the current study is to investigate the perceptions related to health associated with carotenoidbased skin colour, relative to the skin colour conferred by melanin in a group of young Australian adults. It is hypothesized that participants will increase both melanin and carotenoid colouration of facial skin to enhance their perception of a healthy appearance. When colour manipulation is applied in isolation (i.e. two independent, single axes) perceptions of healthiness will be associated with an increased carotenoid colouration and increased melanin colouration. When manipulation of both colours is simultaneously applied (i.e. two-dimensional colour space) perceptions of health will reflect an increased carotenoid colouration and removal of melanin colouration.

\section{Methods}

\section{Ethics statement}

This study obtained ethics approval from the University of Newcastle Human Research Ethics Committee (H-20120405). All participants provided informed consented and all individuals were reimbursed for their participation with a coffee voucher. Additionally, individuals from the School of Nursing and Midwifery research program were eligible to receive five course credit points for their participation.

\section{Participants}

A convenience sample $(n=60)$ was recruited from the University of Newcastle from April-September 2013 through advertisements on notice boards and via a research program in the School of Nursing and Midwifery. Eligible participants were $\geq 18$ years of age, proficient in English, self-reported normal colour vision and able to attend the university campus to complete the computer-based tests. Three participants experienced unexpected error within the computer program during testing and were unable to view the entire set of trial faces. These participants were excluded, leaving 57 participants ( $\mathrm{n}=4$ males, $\mathrm{n}=53$ females) for analysis. Self-reported demographic data (e.g. age, gender, ethnicity), skin colouration type and quality (Fitzpatrick.T.B, 1988), height, weight, general health and fruit and 
vegetable intake was obtained for each participant. The mean $( \pm \mathrm{SD})$ age of participants was $27.9 \pm 7.5$ years with a BMI of $25.6 \pm 7.7 \mathrm{~kg} / \mathrm{m}^{2}$. Seventy-two percent $(\mathrm{n}=41)$ reported their ethnicity as "White Australian" and $43.9 \%(\mathrm{n}=25)$ reported their skin type as "burn easily" and skin quality as "average". Self-reported fruit and vegetable intakes were $1.9 \pm 1.1$ and $2.5 \pm 1.3$ servings per day respectively, and $66.7 \%$ of participants reported not eating adequate fruit and vegetables compared to national recommendations.

\section{Trial face images}

The trial faces A detailed description of the photography methods has been reported elsewhere (Stephen et al., 2011; Stephen et al., 2009; Whitehead, Re, et al., 2012). Standardised photographs of 50 Caucasian adults (25 male: 25 female, mean age 20.5 range $18-27, \mathrm{SD} \pm 1.75$ years) were taken in a grey-coloured booth under standardised lighting conditions, with colour calibration conducted using a Gregtag Macbeth Mini ColourChecker (Pantone). Hair was held back off the face and clothing covered with a grey coloured board to prevent colour reflection.

\section{Melanin and carotenoid axis values}

Existing skin colour measurements (CIE L*a*b* colour space) previously obtained from spectrophotometer readings from human skin were used to derive the melanin and carotenoid skin colour axis values (Stephen et al., 2011; Whitehead, Ozakinci, et al., 2012). Carotenoid values were determined based on the relationship between fruit and vegetable intake and change in skin colour, measured by the mean change in facial colour per additional portion of fruit and vegetables consumed (Stephen et al., 2011; Whitehead, Ozakinci, et al., 2012). This was scaled to a range between 40 to +40 portions of fruit and vegetables per day over a six week period to form the high carotenoid mask at $L^{*}=-4.12$; $\mathrm{a}^{*=5.16} \mathrm{~b}^{*}=15.96$ and the low carotenoid mask at $\mathrm{L}^{*}=4.12 ; \mathrm{a}^{*=}=-5.16 \mathrm{~b}^{*}=-15.96$ (Whitehead, Ozakinci, et al., 2012).

Melanin values were derived by measuring the difference between a sun-exposed region of the outer arm and an unexposed region of the shoulder (Stephen et al., 2011; Whitehead, Ozakinci, et al., 2012). The high melanin mask was set at $L^{*}=-10.40 ; a^{*}=-2.42 b^{*}=14.20$ and the low melanin mask at $L^{*}=10.40 ; a^{*}=2.42 b^{*}=-14.20$. The melanin masks thus gave approximately the same colour difference across the manipulation range $(\Delta \mathrm{E}=17.8)$ as the carotenoid colour $\operatorname{masks}(\Delta \mathrm{E}=17.2)$.

Colour masks for each of the 50 trial face images were developed in MATLAB (using methods described in (Stephen et al., 2011; Whitehead, Ozakinci, et al., 2012) and reflected changes in single carotenoid (b*) and melanin (L*) one- 
dimensional axes. Mean CIEL*a*b* values across skin pixels for each image were calculated using MATLAB to define initial face colour. Thirteen image masks for each single axis were developed and applied to each trial face. The matrix of image masks represented equal increments in colour change (from high to low colour) along a continuum, with the endpoints reflecting maximal colour change (increase and decrease) and the midpoint no change (i.e. original image).

Two-dimensional facial masks were generated by transforming each of the 13 single melanin axis frames along the carotenoid axis (13 frames), consisting of a total of 169 images (13x13).

\section{Colour manipulation experiments}

Each participant completed three computer-based experiments on 50 trial faces "to make the face appear as healthy as possible". In the first two experiments participants independently manipulated the trial face colour along each single axis for carotenoid or melanin by moving the mouse horizontally to select the face frame image with the colour that they perceived as making each face appear the healthiest. In the third experiment, participants manipulated the skin colour along the two-dimensional carotenoid and melanin axes simultaneously, which allowed them to adjust both carotenoid colouration and melanin skin colouration using both horizontal and vertical movements of the mouse. In all three experiments trial faces appeared in random order using the same 50 faces, meaning that participants manipulated the colour for each face three times.

The computer tests were completed in a darkened room with no natural or artificial light, using a laptop with a 17.3 in LCD screen (Dell Inspiron 17R- 5721, Dell Inc., USA). To minimise potential variability introduced by the height and angle of the computer screens, the laptops were placed on laptop stands and the angle of screen set at $\sim 90^{\circ}$ from the base of the stand (i.e. perpendicular). The computer LCD screens were calibrated weekly using Spyder4 Pro (Datacolour, USA).

\section{Data analysis}

Data analysis was undertaken using Stata (Version 11.0; StataCorp, College Station, TX, USA). A significance level was set at 5\%. Univariate ANOVA was used to compare the average colour change in melanin and carotenoid amongst the female and male face trials. One sample t-tests were used to compare overall colour change which was expressed as $\Delta \mathrm{E}$ (Euclidian distance is a standard measure of colour change in the CIE $\mathrm{L}^{*} \mathrm{a}^{*} \mathrm{~b}^{*}$ colour space using the formula 
$\Delta E_{a b}^{*}=\sqrt{\left(L_{2}^{*}-L_{1}^{*}\right)^{2}+\left(a_{2}^{*}-a_{1}^{*}\right)^{2}+\left(b_{2}^{*}-b_{1}^{*}\right)^{2}}$ Linear regression was used to test the associations between starting yellowness $\left(\mathrm{b}^{*}\right)$ of facial trials and melanin and carotenoid preferences.

\section{Results}

\section{Single axis experiment - melanin}

The mean overall colour change preferred in the male face trials was $\Delta \mathrm{E} 0.31(\mathrm{SE} \pm 0.08)$ and for female faces $\Delta \mathrm{E} 0.36$ ( $\mathrm{SE} \pm 0.10)$. There was no main effect of the face trials by sex $(\mathrm{p}=0.72)$.

Figure 1 demonstrates an overall weak effect for melanin colour to be increased by participants. The amount of colour added was significantly associated with initial skin yellowness $\left(b^{*}\right)$ of the trial faces. Participants increased melanin colouration more for those faces that were initially low in yellowness $\left(b^{*}\right)\left(\beta=-0.10, t=-3.83, p<0.001 R^{2}=0.23\right)$.

\section{Single axis experiment - carotenoids}

The mean colour change identified in the male face trials was $\Delta \mathrm{E} 2.41(\mathrm{SE} \pm 0.26)$, equivalent to $5.6 \pm 0.6$ additional servings of fruit and vegetables per day. In female faces it was $\Delta \mathrm{E} 3.20(\mathrm{SE} \pm 0.20)$, which was equivalent to $7.4 \pm 0.6$ additional daily servings of fruit and vegetables. When comparing colour change applied to male and female faces there was a main effect of sex $(F=4.56, p=0.038)$, with more yellowness $\left(b^{*}\right)$ added to female faces. There was a significant preference for increased carotenoid values in both female; $\mathrm{t}(24)=12.30, \mathrm{p}<0.0005$ and male; $\mathrm{t}(23)=8.93$, $\mathrm{p}<0.005$ faces.

Figure 2 illustrates a strong overall preference for higher carotenoid colouration. Participants added carotenoid colouration more to those faces that were initially low in yellowness $\left(b^{*}\right)\left(\beta=-0.386, t=-5.245, p<0.001, R^{2}=0.36\right)$.

\section{Double axis experiment}

One male trial image was excluded from the analysis due to accidental omission, with another male trial viewed twice, therefore the second result from the double axis experiment for that face was excluded leaving $\mathrm{n}=49$ trial faces for analysis. The average carotenoid axis colour change was $\Delta \mathrm{E} 3.15(\mathrm{SE} \pm 0.19)$ and for melanin axis $\Delta \mathrm{E}-1.04(\mathrm{SE} \pm 0.1)$. The average colour change was $\Delta \mathrm{L}^{*}-0.15(\mathrm{SE} \pm 0.04), \Delta \mathrm{a}^{*} 1.09\left(\mathrm{SE} \pm 0.06 ; \Delta \mathrm{b}^{*} 2.09(\mathrm{SE} \pm 0.15)\right.$.

When participants were able to simultaneously manipulate both the level of melanin and the carotenoid colouration, they removed significantly more melanin; $\mathrm{t}(48)=-13.51, \mathrm{p}<0.001$ and added significantly more carotenoid; $\mathrm{t}(48)=16.82$, 
$\mathrm{p}<0.001$ compared to the single axis conditions. The overall colour change indicated a preference for higher facial skin yellowness; $\Delta \mathrm{b}^{*}=+2.09 \pm 0.15, \mathrm{t}(48)=14.05, \mathrm{p}<0.001$ and slightly lowered lightness; $\Delta \mathrm{L}^{*}=-0.15 \pm 0.04, \mathrm{t}(48)=-3.45$, $\mathrm{p}=0.001$.

Figure 3 shows that when both melanin and carotenoid colouration were able to be manipulated simultaneously there was a significantly higher change in carotenoid colouration $(\Delta \mathrm{E}=3.15)$ relative to melanin $(\Delta \mathrm{E}=-1.04)$.

\section{Discussion}

The current study demonstrates that perceptions of the appearance of health of a group of facial images of young adults, by Australian adults is related to skin colouration contributed by higher carotenoid content (skin yellowness) and decreased melanin content (skin tanning). When the two colours were manipulated independently in the single axis experiments, in both conditions adults evaluating the facial images significantly increased melanin colouration and increased skin carotenoid colouration. When both carotenoid and melanin colouration were manipulated simultaneously in the facial images, participants chose to increase carotenoid colouration and remove melanin colouration in order to make the faces appear the most healthy. While both pigments contribute to a rise in the appearance of skin yellowness, higher melanin induces a reduction in overall skin lightness. Of interest was that participants increased both carotenoid and melanin colour more in the facial images that were initially low in skin yellowness $\left(b^{*}\right)$. This suggests that skin colouration that is of low yellowness is viewed by other adults as appearing less healthy compared to higher levels of yellowness.

Our findings from the single carotenoid and melanin axes manipulations are consistent with previous studies conducted with Caucasian adults in Scotland (Stephen et al., 2011; Whitehead, Ozakinci, et al., 2012). In those studies, participants added relatively higher amounts of carotenoid based colour and smaller amounts of melanin to face images (Stephen et al., 2011). When Scottish participants simultaneously manipulated both the colour axes the results diverged from the Australian participants. Scottish participants like Australian participants increased carotenoid colouration more than melanin. However, Australian participants removed melanin colouration whereas Scottish participants significantly increased melanin based colour (Stephen et al., 2011; Whitehead, Ozakinci, et al., 2012). This suggests that Scottish adults associate a higher level of skin melanin pigmentation as conferring a healthy appearance compared to Australian adults. This finding could potentially be due to cultural differences in relation to tanning between the two groups and an 
increased awareness of the associated dangers of prolonged sun exposure likely to be greater in Australia due to increased risk of skin cancer compared to Scotland (Hamilton et al., 2012).

In the current study we found that carotenoid colouration was preferred more in female faces compared to male faces. These findings have also recently been reported from a study with a group of Scottish adults who have examined attractiveness in facial images (Lefevre, 2015). There were no sex differences in preferences for melanin colouration however preferences for melanin were significantly higher in male faces for attractiveness compared to female faces (Lefevre, 2015).

This current study has several limitations. The majority (93\%) of participants in the current study were females thus limiting the ability to generalise the findings to Australian men. In studies conducted using Scottish participants, only $38-45 \%$ of the subjects were male. The majority of participants were also nursing students (\%) which may be more health conscious or perceive health differently when compared to the general population.

A further limitation may relate to the effects of using different facial identities or computer monitors as previous studies used cathode ray tube monitors, which may explain some of these differences. However, to minimise any potential effect of the display device, the monitors were regularly colour calibrated and setup in a standardized way with the screen viewed at a standardized angle.

\section{Conclusion}

In summary, Australians perceive facial skin colouration associated with both carotenoid intake from fruit and vegetables and melanin due to sun exposure as conveying the appearance of health in young adults. However, colouration due to carotenoids was preferred over melanin as a contribution to the appearance of health in young adult facial images. Further research using Australian facial images and rating performed by male participants is warranted in order to evaluate these relationships in the population more broadly. 


\section{References}

Alaluf, S., Heinrich, U., Stahl, W., Tronnier, H., \& Wiseman, S. (2002). Dietary Carotenoids Contribute to Normal Skin Colour and UV Photosensitivity. The Journal of Nutrition, 132, 399-403.

Australian Bureau of Statistics. (2014). 4364.0.55.007 - Australian Health Survey: Nutrition First Results - Foods and Nutrients, 2011-12 Canberra: ABS.

Fitzpatrick.T.B. (1988). The Validity and Practicality of Sun-Reactive Skin Types I Through VI. JAMA Dermatology, 124(6), 869-871. doi: doi:10.1001/archderm.1988.01670060015008

Hamilton, K., White, K. M., McD. Young, R., Hawkes, A. L., Starfelt, L. C., \& Leske, S. (2012). Identifying critical sun-protective beliefs among Australian adults. Health Education Research, 27(5), 834-843. doi: 10.1093/her/cys093

Lefevre, C. E. P., D.I. (2015). Fruit over sunbed: Carotenoid skin colouration is found more attractive than melanin colouration. Q J Exp Psychol, 68(2), 284-293. doi: 10.1080/17470218.2014.944194

Mayne, S. T., Cartmel, B., Scarmo, S., Lin, H. Q., Leffell, D. J., Welch, E., . . Gellermann, W. (2010). Noninvasive assessment of dermal carotenoids as a biomarker of fruit and vegetable intake. American Journal of Clinical Nutrition, 92(4), 794-800. doi: 10.3945/ajen.2010.29707

Pezdirc, K., Hutchesson, M., Whitehead, R., Ozakinci, G., Perrett, D., \& Collins , C. (2015). Fruit, Vegetable and Dietary Carotenoid Intakes Explain Variation in Skin-Color in Young Caucasian Women: a Cross-Sectional Study. Nutrients, 7(7), 5800-5815. doi: 10.3390/nu7075251

Stephen, I. D., Coetzee, V., \& Perrett, D. I. (2011). Carotenoid and melanin pigment coloration affect perceived human health. Evolution and Human Behavior, 32(3), 216-227. doi: 10.1016/j.evolhumbehav.2010.09.003

Stephen, I. D., Law Smith, M. J., Stirrat, M. R., \& Perrett, D. (2009). Facial Skin Coloration Affects Perceived Health of Human Faces. Int J Primatol, 30, 845-857. doi: 10.1007/s10764-009-9380-z

Whitehead, R., Ozakinci, G., \& Perrett, D. (2012). Attractive Skin Coloration: Harnessing Sexual Selection to Improve Diet and Health. Evolutionary Psychology, 10(5), 842-854. 
Whitehead, R., Re, D., Xiao, D., Ozakinci, G., \& Perrett, D. (2012). You are what you eat: Within-subject increases in fruit and vegetable consumption confer beneficial skin-color changes. PLoS ONE, 7(3). doi: 10.1371/journal.pone.0032988 
Figure Legends:

Figure 1 Preferences for skin colour associated with melanin (single axis melanin experiment). The dashed line represents the line of best fit between initial skin yellowness $\left(b^{*}\right)$ and change in melanin $(\Delta \mathrm{E}) . \Delta \mathrm{E}$ is a standard way of representing colour differences in CIE $\mathrm{L}^{*} \mathrm{a}^{*} \mathrm{~b} *$ colour space and here represents the melanin colour change applied to the original photographs. $\mathrm{B}^{*}$ reflects degree of skin yellowness and in this figure represents the amount of skin yellowness in the original face images.

Figure 2 Preferences for skin colour associated with dietary fruit and vegetable intake (single axis carotenoid experiment). The facial images on the left reflect high fruit and vegetable colouration (top image) and low fruit and vegetable colouration (bottom image). The dashed line in the graph displays the best-fit regression line between initial skin yellowness $\left(b^{*}\right)$ and the colour change $\Delta \mathrm{E}$ (right axis) along with colour change in terms of fruit and vegetable portions (left axis).

Figure 3 Colour change applied simultaneously along the carotenoid axis (associated with fruit and vegetable intake) and melanin axis to the original face images (double axis experiment). On average, there was a significantly higher change in carotenoid colouration $(\Delta \mathrm{E}=+3.15)$ compared with melanin colouration $(\Delta \mathrm{E}=-1.04)$. 


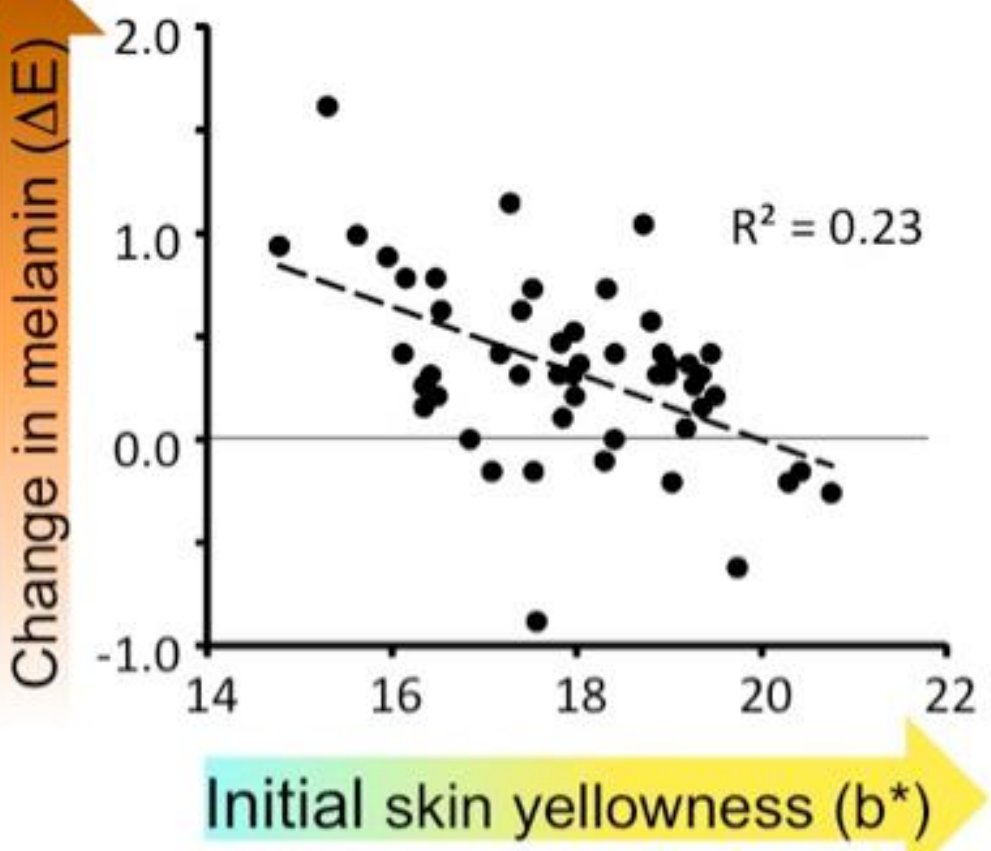

Figure 1 

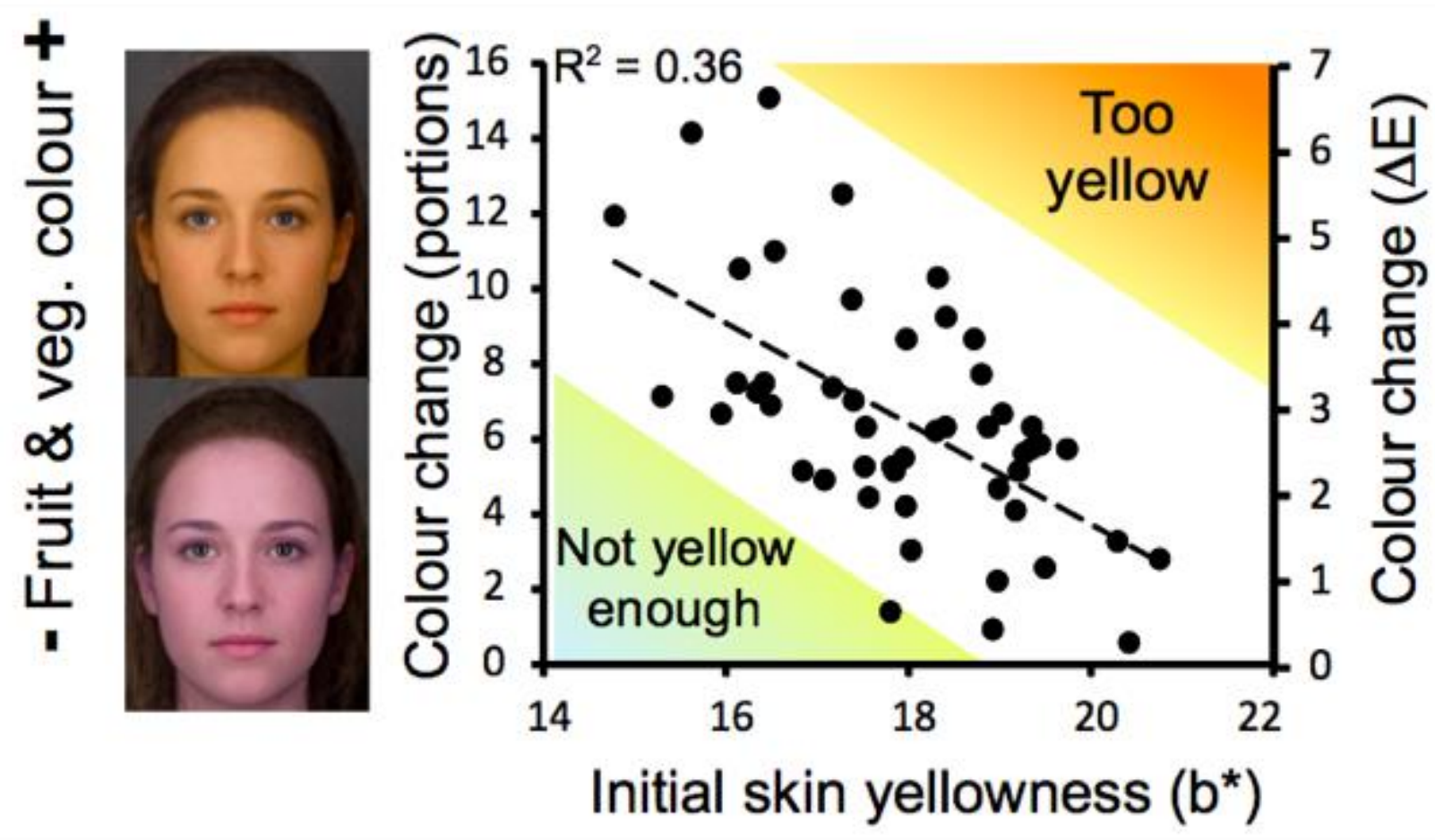

Figure 2 


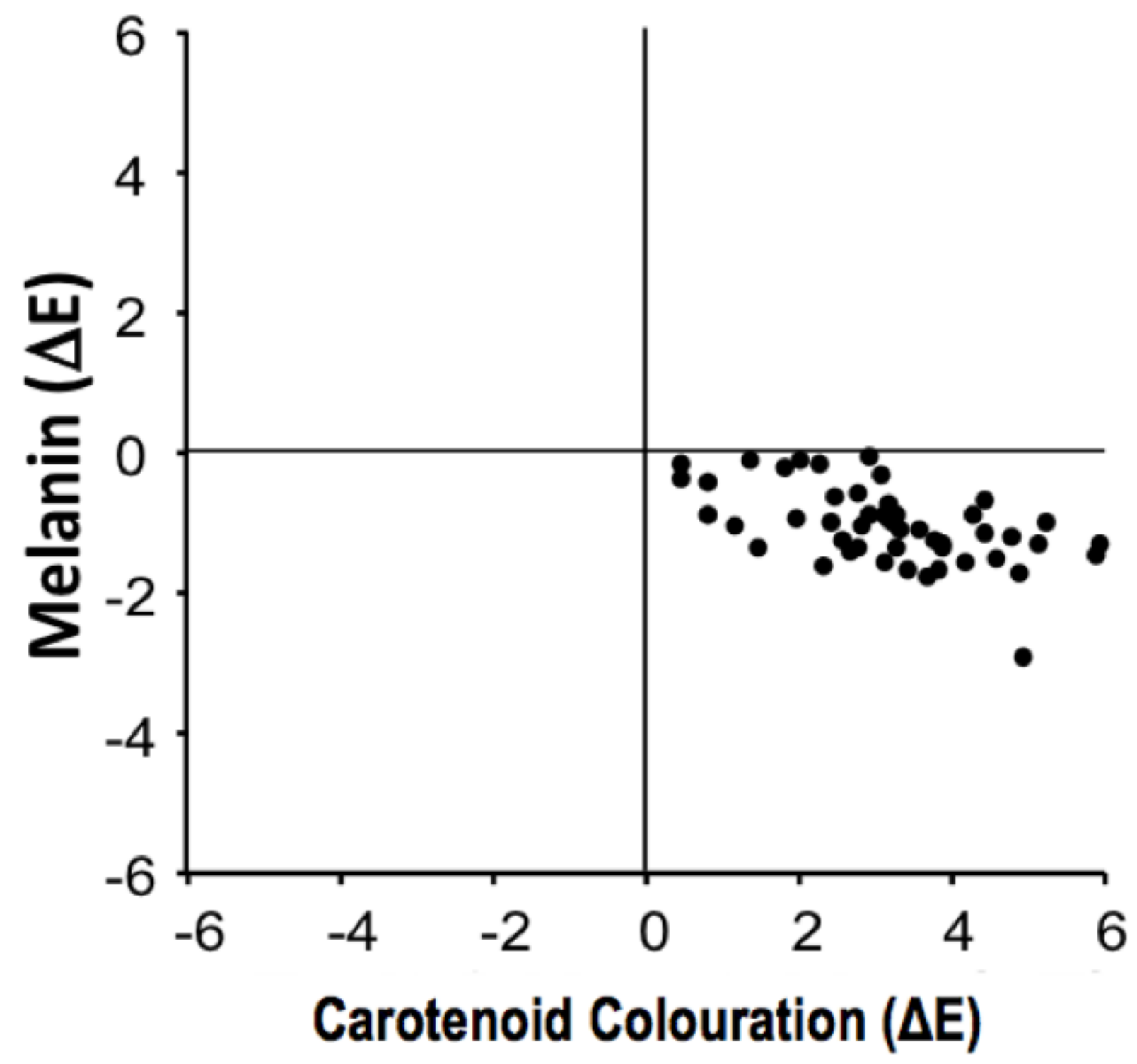

Figure 3 